\title{
Economía social y solidaria en el espacio eurolatinoamericano. Innovación social para la internacionalización de cooperativas ${ }^{1}$
}

\author{
Social and Solidary Economy in the Euro-Latin American Space. \\ Social Innovation for the Internationalization of Cooperatives
}

César SÁnchez Álvarez

\section{Resumen}

El artículo se centra en el concepto dual de la economía social y solidaria, con el objetivo de presentar las características del sector en Colombia y en Europa, y así establecer puntos comunes y de interés para su fortalecimiento en el espacio eurolatinoamericano. Se detalla la importancia de la ESS para el desarrollo social y el papel de las alianzas intercooperativas como elemento de innovación social, identificando los elementos de mayor importancia para fomentar el modelo basado en la pluralidad y la democracia económica que contribuye al desarrollo sostenible, además de fomentar el tejido asociativo y la igualdad de oportunidades en las dos orillas del Atlántico.

Palabras clave: alianzas público-privadas, cooperativas, economía social y solidaria, innovación social, internacionalización.

\begin{abstract}
The paper focuses on the dual concept of social and solidarity economy, in order to present the characteristics of the sector in Colombia and Europe, and thus establish common and interest points for its strengthening in the Euro-Latin American Space. It details the importance of the ESS (social and solidarity economy) for social development and the role of inter-cooperative alliances as an element of social innovation, identifying the most important elements to promote the model based on plurality and economic democracy that contributes to sustainable development, as well as promote associative tissue and equal opportunities on both sides of the Atlantic.
\end{abstract}

Keywords: public-private partnerships, cooperatives, social and solidarity economy, social innovation, internationalization.

\footnotetext{
1 Este artículo fue el ganador del I puesto en el Concurso de Investigación Rymel Serrano Uribe, 2012. Como incentivo a la difusión de la producción intelectual, Cooperativismo y Desarrollo aunó esfuerzos con la Universidad Cooperativa de Colombia para divulgar, luego de un arduo proceso de selección y arbitraje, los mejores artículos presentados. Pese a su presentación en concurso, este artículo es de índole inédita en el campo de la divulgación científica.

- Candidato a doctor en Economía Social y Cooperativa por la Universidad de Valencia. Becario del Ministerio de Asuntos Exteriores y Cooperación -AECID en el IER- de la Pontificia Universidad Javeriana. Correo electrónico: csa.puj@gmail.com
}

Cómo citar este artículo: Sánchez Álvarez, C. (2012). Economía social y solidaria en el espacio eurolatinoamericano. Innovación social para la internacionalización de cooperativas. Revista Cooperativismo \& Desarrollo, 20(101), 190-212. 
Descriptores alfanuméricos (JEL codes): L330-Comparison of Public and Private Enterprises and Nonprofit Institutions; Privatization; Contracting Out; L380-Public Policy; P130-Cooperative Enterprises; P590-Comparative Economic Systems: Other.

Recibido: 15 de agosto del 2012 Aprobado: 15 de septiembre del 2012

\section{Introducción}

El fenómeno social y económico objeto de este artículo tiene diferentes aproximaciones conceptuales que comparten diferentes puntos en común a nivel internacional. Es importante subrayar que las conceptualizaciones dependen de las características del contexto institucional económico del país o de los territorios que comparten cultura y políticas de integración. El presente trabajo se fundamenta en el común denominador que existe en todos los países, aunque hayan alcanzado niveles de desarrollo económico y social. Con esto entendemos que existen problemas comunes a todos los países, como subrayó el profesor Rymel Serrano durante el discurso de inauguración de la Primera Conferencia Internacional del Ciriec-Colombia. ${ }^{2}$ Según el profesor Rymel Serrano, los individuos responden a estos problemas con formas comunes dentro del espectro del asociacionismo y la autogestión. Es decir, a través de la democratización económica, intentan dar y estructurar respuestas, siendo esto la característica principal para generar ciudadanía participativa.

\footnotetext{
Discurso inaugural del profesor Rymel Serrano durante la I Conferencia Internacional del Ciriec-Colombia. Se celebró el 29 de marzo del 2012 en la sede de la Universidad Cooperativa de Colombia su primer Congreso bajo el lema de "Tendencias en la gestión de la economía pública y social".
}

La necesidad de tratar el tema de la economía social y solidaria se justifica por el contexto internacional actual. En primer lugar, la trayectoria argumentativa que legitima a nivel internacional la economía social y solidaria y el movimiento socioeconómico punta de diamante: el movimiento cooperativista.

Las Naciones Unidas declararon el 2012 como el Año Internacional de las Cooperativas, reconociendo el movimiento cooperativista como elemento de desarrollo social, económico, cultural y ambiental, que favorece el progreso humano y da respuesta a los cambios que las tendencias sociales y económicas condicionan en cuanto a la transformación social (Sánchez, 2011).

En segundo lugar, la situación actual que vive el modelo económico predominante, que ve en la economía neoliberal su máxima referencia teórica y la empresa capitalista como organización y coordinación de los factores de producción. Se hace necesario proponer y formular nuevas formas de hacer empresa, además de entender y dar elementos que fundamenten nuevas formas de relaciones económicas centradas en las personas.

\section{Metodología}

El artículo se centra en el concepto dual de la economía social y solidaria (ESs). El objetivo es presentar las características del fenómeno en Colombia 
y en Europa, dando la posibilidad de establecer puntos comunes y de interés para su fortalecimiento en el espacio eurolatinoamericano.

Para alcanzar el objetivo propuesto, el artículo realiza una aproximación a los rasgos cuantitativos y cualitativos más importantes de los últimos años, además de identificar los cambios significativos del sector en relación con el reconocimiento por las instituciones públicas.

Con el fin de definir la importancia de la Ess en el desarrollo social y el papel de las alianzas intercooperativas e internacionales, se identificarán los elementos de mayor importancia para fomentar el modelo basado en la pluralidad y la democracia económica que contribuye al desarrollo sostenible. Asimismo, se identifican las características fundamentales para fomentar el tejido asociativo y la igualdad de oportunidades en las dos orillas del Atlántico. Por tanto, se realiza una revisión de elementos relevantes que indican el proceso de fomento de la Ess, además de considerar los componentes actuales que argumentan a favor de la Ess en el contexto territorial de estudio: el espacio eurolatinoamericano.

\section{Marco teórico de referencia. Enfoques dentro de la economía social y solidaria}

La economía social y solidaria es un concepto dual que indica dos grandes campos de estudio del mismo fenómeno socioeconómico, cultural y político que se da en contextos geográficos diversos, a saber: en el caso de la economía social, en el contexto europeo, y en el caso de la economía solidaria, en el latinoamericano.

La economía social está compuesta por organizaciones económicas cuya ética traduce los siguientes principios: finalidad de servicio a los miembros o a la colectividad más que de beneficio, autonomía de gestión, procesos de decisión democrática, primacía de las personas y del trabajo sobre el capital en el reparto de beneficios (Monzón y Defourny, 1992).

Igualmente, las empresas de economía solidaria se caracterizan por la actividad económica basada en la asociación voluntaria, la propiedad común de los medios de producción, la gestión colectiva, el poder ejercido por la comunidad de trabajadores y en el esfuerzo mutuo, en pro de intereses comunes (Verano, citado en Gaiger, 2009).

Por tanto, en una primera aproximación para definir la ESs, podemos identificar que está compuesta por empresas sin ánimo de lucro que producen bienes o servicios, operantes bajo formas asociativas y de propiedad común, cuyo objetivo es satisfacer las necesidades de sus miembros y de la comunidad.

En el caso de Colombia, las organizaciones de la economía solidaria principalmente son: cooperativas, mutualidades, asociaciones y fondos de empleados. A su vez, en el contexto europeo, las organizaciones que caracterizan la economía social son: cooperativas, mutuas $\mathrm{y}$ asociaciones.

La concepción de la economía social y solidaria según Mutuberría y Serrano 
(2010) pertenece a dos enfoques teóricos. En un primer enfoque se entiende como una solución dentro del sistema capitalista, englobando tres propuestas: (I) políticas y programas de alivio a la pobreza; (II) sistema de garantías con el objetivo de reconstruir espacios propios del Estado de Bienestar, y (III) fomento y conformación de cooperativas. En el caso del segundo enfoque se considera la función transformadora, que es la forma alternativa al sistema capitalista.

Los dos enfoques anteriores caracterizan la complejidad del concepto y su dualidad, es decir, el enfoque en los países centrales de una economía social tiene como objeto las necesidades que se presentan ante los fallos del Estado y los fallos del mercado, teniendo una relación de complementariedad con la economía capitalista. En cambio, en el caso de la economía solidaria, tienen su fuerza en la capacidad de desarrollar prácticas de organización del trabajo, producción, distribución, consumo y acumulación, alternativas al capitalismo, teniendo de esta forma una acción crítica al sistema.

Asimismo, podemos encontrar en la literatura anglosajona el concepto de tercer sector, que se distingue por las siguientes características: organización formal, sector privado, independencia, no reparto de beneficios, voluntariedad de los recursos (Salamon y Anheier, 1997).

La existencia de diferentes conceptos es un claro indicador de la complejidad del fenómeno, que en parte intentan definir la realidad y en parte crear un contenedor donde se incluyan los diferentes elementos que caracterizan estilos de relaciones económicas diversas a las promovidas por el capitalismo en la economía de mercado.

Las características de la Ess se combinan en economías de mercado generando nuevas alternativas a la hegemonía de la centralidad del factor capital en las funciones productivas, ofreciendo una dimensión totalmente diversa, es decir, la centralidad del factor productivo de trabajo y con ello el elemento humano, en el que la persona está en el centro de la actividad económica. Sin entrar en mayor detalle, la Ess, al poner en el centro la persona de la actividad económica, genera externalidades de importancia vital para los sistemas sociales, en especial modo, la capacidad para fortalecer bienes intangibles, de difícil medición cuantitativa, como son la confianza, la reputación, el capital social, la felicidad pública, el civismo, entre otros, que pertenecen a la economía civil (Bruni y Zamagni, 2004), en la que la sociedad civil organizada tiene una gran importancia en la promoción, la formación y el fortalecimiento de formas asociativas de empresas.

\section{Dimensiones de la economía solidaria en Colombia}

La economía solidaria en Colombia es considerada como la intersección entre el tercer sector - constituido por instituciones privadas no gubernamentales, con formas propias de autogobierno y que se crean voluntariamente-, el sector privado empresarial y un segmento del sector estatal (DNP, 2010). 
La evidencia colombiana demuestra que a pesar de diferentes intentos aislados, tanto por parte del gobierno como por parte de las mismas cooperativas y sus gremios, las políticas públicas hacia el sector no se han incluido en la agenda pública nacional ni se han convertido en una políticas de Estado (Castillo et al., 2011).

El fomento de la economía solidaria a través de normas tiene su inicio en Colombia en 1931, con la promulgación de la primera ley de cooperativas (Ley 134 de 1931). ${ }^{3}$ Con el Decreto 2536 de 1986 se institucionaliza el concepto de economía solidaria, definiéndose como "sector conformado por un franja de organizaciones de tipo participativo abierto y otras formas asociativas [...] de un amplio criterio democrático participativo [...]" (DNP, 2010, s.p.). El mismo decreto crea el Consejo Nacional de Economía Solidaria, reconociéndose como un sector de gran importancia para la economía nacional.

Con la Ley 79 del 1988 se da forma al cuerpo de organizaciones solidarias, a saber, cooperativas, asociaciones mutuales y fondos de empleados. Es a partir de 1999 que se evidencia un importante desarrollo de entidades; sin embargo, la falta de un marco regulador propició la informalidad de la actividad solidaria. Producto de esta crisis, se expidió la Ley 454 de 1998, que transformó el marco institucional-regulador e identificó otras organizaciones como son las asociaciones y grupos de voluntariado, corporaciones,

Para un análisis exhaustivo de la historia de las políticas públicas del sector en Colombia se recomienda ver Castillo et al., 2011. fundaciones, juntas de acción comunal y cajas de compensación familiar.

Asimismo, la Constitución de Colombia de 1991 subraya en sus artículos 58, 68 y $333,{ }^{4}$ la importancia de las "organizaciones solidarias" para el desarrollo de la comunidad, lo que implicó un esfuerzo por parte del Estado en soportar el sector y promover sus formas de organizaciones asociativas y solidarias.

En el artículo 4 de la Ley 79 de 1988, el legislador define la empresa cooperativa como una

[...] empresa asociativa, sin ánimo de lucro, en la cual los trabajadores o los usuarios, son simultáneamente los aportantes y los gestores de la empresa, creada con el objeto de producir o distribuir conjuntamente y eficientemente bienes o servicios para satisfacer las necesidades de sus asociados y de la comunidad en general [...] (Congreso de Colombia, 1988).

Igualmente, la Ley 454 de 1998 recoge una definición más incluyente de las organizaciones solidarias; en concreto, en su artículo 6-2, se establecen las grandes familias de las organizaciones de la economía solidaria. ${ }^{5}$ Además de

\footnotetext{
4 La Carta Magna respalda las organizaciones solidarias y las formas asociativas y solidarias de propiedad encuentran pleno reconocimiento constitucional como se desprende de lo dispuesto en el Preámbulo y los artículos $1,38,51,57,58,60,64,103,189-24$ y 333.

5 Art. 6-2 de la Ley 454 de 1998: “[...] las cooperativas, los organismos de segundo y tercer grado que agrupen cooperativas $u$ otras formas asociativas y solidarias de propiedad, las instituciones auxiliares de la economía solidaria, [...], las empresas solidarias de salud, las precooperativas, los fondos de empleados, las asociaciones mutualistas, las empresas de servicios en las formas de administraciones públicas cooperativas $[\ldots]$, y todas aquellas formas asociativas solidarias que cumplan las características mencionadas en el presente capítulo" (Congreso de Colombia, 1998, s.p.).
} 
las normas anteriormente indicadas, el sector está regulado por normas específicas: Decreto-ley 1480 de 1989 sobre asociaciones mutualistas, Decreto-ley 1481 de 1989 sobre Fondos de Empleados y Decreto-ley 1482 de 1989 sobre administraciones publicas cooperativas.

Para el objetivo del presente artículo, y para tener un cuadro claro del sector, a continuación se subrayan los datos más relevantes de este, considerando los tres grandes grupos de la economía solidaria: cooperativas, cuya presencia es cuantitativamente la más importante dentro del sector de la Ess, los fondos de empleados y las asociaciones mutuales.

Según los datos más actualizados de Confecoop, en el 2011 (Confecoop, 2012) se observa la situación que se presenta en la tabla 1 .

Tabla 1. Universo organizacional de la economía solidaria en Colombia

\begin{tabular}{l|c|c|c}
\hline \multicolumn{1}{c|}{ Naturaleza } & $\mathbf{N}^{\mathbf{o}}$ entidades & $\mathbf{N}^{\mathbf{o}}$ asociados & $\mathbf{N}^{\mathbf{o}}$ empleados \\
\hline Cooperativas & 7.848 & 5.384 .133 & 138.549 \\
\hline Fondos de empleados & 2.102 & 922.239 & 5.532 \\
\hline Asociaciones mutuales & 292 & 213.134 & 4.890 \\
\hline Total & 10.242 & 6.519 .506 & 148.971 \\
\hline
\end{tabular}

Fuente: el autor, a partir datos Confecoop (2012)

Es interesante subrayar el andamiento del sector en los últimos diez años en Colombia. Según los datos recogidos por Confecoop, en el periodo 2000-2011 el sector solidario colombiano a nivel nacional ha evolucionado de la siguiente forma, como se puede observar en la tabla 2 .

Desde el 2000 se denota un neto incremento del número de entidades del sector de la economía solidaria, al cual le corresponde un aumento de los activos y un patrimonio que se incrementa cada año; este último se queda por encima de los nueve mil millones de pesos en el 2011.

Un dato interesante es el número de asociados que, después de un decremento interanual entre el 2000 y el 2001, vuelve a crecer para registrarse un total de 5.384.133 asociados en el 2011.

En el curso de los últimos años, el fenómeno ha aumentado el número de trabajadores empleados: desde el 2000 el número de empleados ha sido más que duplicado, pasando de ser de un total de 51.085 en el 2000, a un total de 138.549 en el 2011. Esto indica un incremento del $171 \%$, con una media de incremento interanual del 9,78\% - lo cual podría ser interpretado como el porcentaje de nuevos trabajos generados por la economía solidaria-, registrándose sólo en dos ocasiones un decremento interanual: del 2,59\% entre el 2006 y el 2007, y del $0,83 \%$ entre el 2010 y el 2011. 
Tabla 2. Principales dimensiones de la economía solidaria en Colombia (2000-2011)

\begin{tabular}{c|c|c|c|c|c|c|c|c}
\hline Año & $\begin{array}{c}\mathbf{N}^{\mathbf{0}} \\
\text { enti- } \\
\text { dades }\end{array}$ & Activos* & Pasivos* & $\begin{array}{c}\text { Patrimo- } \\
\text { nio* }^{*}\end{array}$ & Ingresos* & $\begin{array}{c}\text { Exce- } \\
\text { dentes* }\end{array}$ & Asociados & $\begin{array}{c}\text { Emplea- } \\
\text { dos }\end{array}$ \\
\hline 2000 & 3.472 & 4.772 .886 & 2.466 .781 & 2.306 .105 & 5.790 .995 & 180.401 & 2.439 .122 & 51.085 \\
\hline 2001 & 3.517 & 6.034 .635 & 3.476 .196 & 2.558 .440 & 6.628 .317 & 220.866 & 1.617 .898 & 55.530 \\
\hline 2002 & 4.195 & 8.174 .549 & 4.868 .028 & 3.306 .521 & 9.357 .078 & 264.639 & 2.430 .649 & 69.030 \\
\hline 2003 & 5.107 & 9.492 .518 & 5.650 .645 & 3.841 .873 & 11.557 .137 & 305.381 & 2.602 .146 & 80.987 \\
\hline 2004 & 5.931 & 10.755 .077 & 6.326 .423 & 4.431 .450 & 13.198 .504 & 292.786 & 3.024 .101 & 93.945 \\
\hline 2005 & 6.462 & 12.467 .475 & 7.406 .841 & 5.066 .830 & 14.945 .484 & 294.540 & 3.305 .433 & 107.986 \\
\hline 2006 & 6.877 & 13.857 .713 & 8.080 .137 & 5.777 .576 & 17.205 .682 & 349.919 & 3.682 .496 & 114.924 \\
\hline 2007 & 7.349 & 16.871 .647 & 10.255 .902 & 6.615 .896 & 20.230 .356 & 373.914 & 4.020 .334 & 111.951 \\
\hline 2008 & 7.833 & 19.372 .178 & 11.722 .624 & 7.649 .554 & 22.583 .734 & 393.217 & 4.473 .514 & 123.643 \\
\hline 2009 & 8.124 & 21.200 .500 & 12.856 .786 & 8.343 .715 & 24.529 .649 & 384.487 & 4.821 .763 & 137.888 \\
\hline 2010 & 8.533 & 24.162 .013 & 14.915 .496 & 9.246 .645 & 27.963 .580 & 404.133 & 5.131 .780 & 139.703 \\
\hline 2011 & 7.848 & 24.186 .004 & 14.330 .748 & 9.855 .400 & 27.991 .478 & 370.925 & 5.384 .133 & 138.549 \\
\hline
\end{tabular}

* Cifras monetarias en millones de pesos.

Fuentes: SIAC Confecoop, Superfinanciera, Supervigilancia, Superservicios, Saludcoop, Acemi, Gestar Salud. Elaboración del autor a partir de datos tomados de las bases de datos de la Superintendencia de la Economía Solidaria en Colombia

En las figuras 1, 2 y 3 se observa la de entidades y número de asociados, evolución positiva de los datos más relerespectivamente.

vantes: número de empleados, número

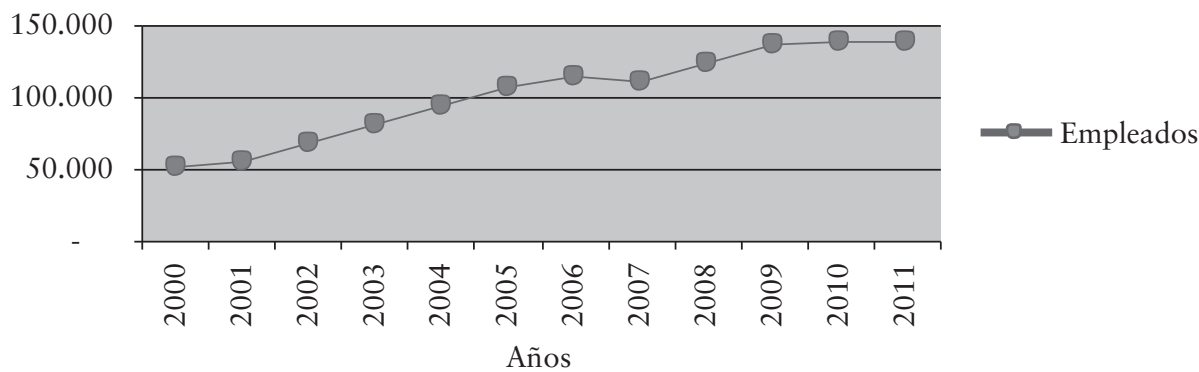

Figura 1. Evolución del número de empleados en el sector solidario colombiano

Fuente: el autor 


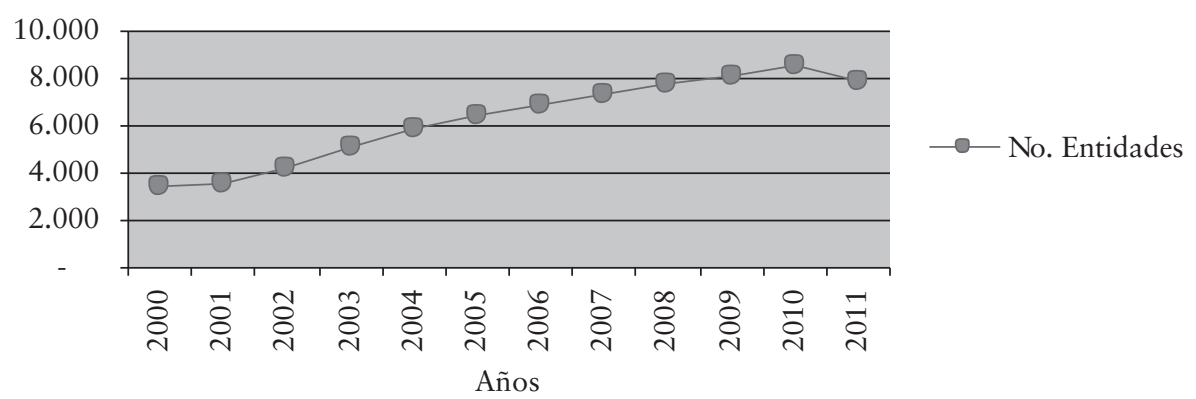

Figura 2. Evolución del número de entidades en el sector solidario colombiano Fuente: el autor

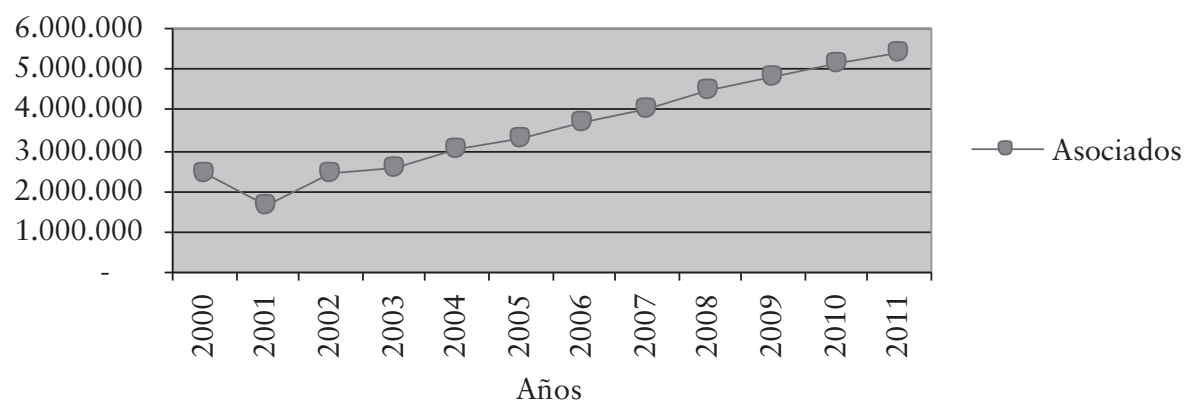

Figura 3. Evolución del número de asociados en el sector solidario colombiano

Fuente: el autor

Por orden de magnitud, los principales sectores en los que las cooperativas operan son los siguientes: trabajo asociado, aporte y crédito, transporte, agropecuario y financiero, educación y salud. Dichas entidades están distribuidas en 32 departamentos y en 640 municipios, y son mayoritariamente micro y pequeñas dimensiones.

Se ha registrado un descenso interanual en el número de las cooperativas con respecto al $2010(-5,7 \%)$, debido a las medidas tomadas por el Gobierno dirigidas a combatir y eliminar las cooperativas de trabajo asociados que no cumplían con los principios centrales del modelo. Es importante destacar cómo, además de la creación de empleo —las personas empleadas en la cooperativas representan el $3,43 \%$ de la población económica activa del país y el 3,96\% de la población ocupada (Confecoop, 2012)-, las cooperativas contribuyen al PIB con sus ingresos en una proporción 
del 4,7\%. Por tanto, el cuadro delineado del fenómeno confirma la importancia de considerar las organizaciones solidarias como actores importantes en los planes de desarrollo de Colombia.

\section{Evolución y legitimación de la economía social en Europa}

La realidad europea es bastante rica en contribuciones al debate sobre la economía social y los elementos que la identifican y caracterizan. Desde un punto de vista político europeo, la economía social se pone dentro de la estrategia del Tratado de Lisboa; específicamente, el Tratado de la Unión Europea (TUE) prevé la concepción de economía social de mercado altamente competitiva dirigida al pleno empleo y al progreso social, favoreciendo el desarrollo de Europa (artículo 3.3, TUE).

La importancia de la economía social en la Unión Europea ha ido aumentando según las políticas que han caracterizado el proceso de creación de la Unión Europea con 27 países en torno a

[...] los valores de respeto de la dignidad humana, libertad, democracia, igualdad, Estado de Derecho y respeto de los derechos humanos, incluidos los derechos de las personas pertenecientes a minorías. Estos valores son comunes a los Estados miembros en una sociedad caracterizada por el pluralismo, la no discriminación, la tolerancia, la justicia, la solidaridad y la igualdad entre mujeres y hombres (TUE, Artículo 1bis, s.p.).

Asimismo, podemos encontrar diferentes resoluciones del Parlamento Euro- peo, directivas del Consejo y Parlamento Europeo, comunicaciones de la Comisión y dictámenes del Comité Económico y Social que indican la sintonía con los asuntos de las entidades de economía social europea. En concreto, el "Informe sobre la situación de la Economía Social en Europa" ${ }^{\prime}$ ha demostrado la importancia de la economía social en la economía de la UE. El informe establece diferentes conclusiones que corresponden a la situación actual de un sector que desarrolla acciones y obtiene resultados coherentes a los principios y valores de la Unión Europea y postula la creciente importancia de la economía social de mercado y de no mercado en el tejido socioeconómico.

Históricamente en Europa las organizaciones de economía social se han relacionado directamente con asociaciones populares y con cooperativas, constituyendo la espina dorsal de la economía social europea. El sistema de valores y los principios de las -históricasasociaciones populares han sido sintetizados por el movimiento cooperativo histórico y son los elementos utilizados para formular el concepto moderno de economía social, estructurado alrededor de cooperativas, mutuas, asociaciones y fundaciones (Chaves y Monzón, 2007).

Los números de las organizaciones de economía social son bastante significativos. En el 2005 existían más de

\footnotetext{
6 Conocido como Informe Chaves-Monzón. Presentado al Consejo Económico y Social Europeo el 5 de diciembre del 2007.
} 
240.000 cooperativas que desarrollaban actividades económicas en la Unión Europea, con 25 países. Se trata de empresas que están radicadas en todos los sectores de relevancia económica, bien sea por los servicios o los bienes que producen. Por ejemplo, las cooperativas dan directamente trabajo a 37 millones de personas y reúnen 143 millones de socios. Asimismo, en 1997 en la UE con 15 países, las asociaciones daban trabajo a 6,3 millones de personas y en el 2005 en la UE-25, representaban más del $4 \%$ del producto interno bruto, reagrupando el $50 \%$ de los ciudadanos de la Unión Europea. En el caso de las fundaciones, en el 2000 se contabilizaban más de 75.000 fundaciones, número en fase de crecimiento desde los años ochenta en los 25 estados miembros, incluidos los países de Europa Central y Oriental, dando trabajo a más de cinco millones de voluntarios (Chaves y Monzón, 2007).

Ante los datos que presenta el Informe, nos encontramos la importancia creciente en las últimas décadas. La importancia no se debe solamente a los datos cuantitativos en el empleo respecto al empleo total (véase tabla 3), sino también de su capacidad para contribuir al bienestar.

En Europa las organizaciones de economía social se han consolidado como organizaciones necesarias para dar estabilidad y sostenibilidad al crecimiento económico, sobre todo por la capacidad de generar participación en la producción de riqueza - dentro del mercado-, siendo complementarias a las empresas capitalistas y por su eficacia en soluciones a nuevos problemas sociales -fuera del mercado. En síntesis, se puede decir que son organizaciones que actúan paliando las externalidades negativas producidas por el sistema económico capitalista y complementando las acción social del Estado.

Las características que acomunan las entidades de la economía social son su composición de pluralidad, diversidad. Una de las características más relevantes es el motivo de su creación: orientado a satisfacer y resolver necesidades de personas, hogares o familias pertenecientes a muy diversos colectivos sociales.

Los intereses de las personas convergen en diversos papeles dentro y fuera de la actividad empresarial, y específicamente las organizaciones de economía social consiguen encontrar equilibrios en la competitividad de los mercados, como es el caso de las cooperativas de consumo y trabajo y las organizaciones de economía social de segundo grado. Las anteriores organizaciones agregan diversas organizaciones de economía social con intereses que son a menudo causas de conflicto y competitividad del sector.

Otra característica que se puede encontrar en estas organizaciones es que consiguen canalizar el conflicto de intereses y definir el conflicto de identidad para establecer una estructura de preferencias razonables entre los sujetos que participan democráticamente. Por tanto, si las "preferencias de las personas ayudan a determinar qué organizaciones e instituciones se perpetúan" (Ben-Ner y Putterman, 1999, p. 57), podemos 
Tabla 3. Empleos remunerados en la es comparado con el empleo remunerado total. Unión Europea (2002-2003)

\begin{tabular}{|c|c|c|c|c|c|c|c|}
\hline Ranking & País & $\begin{array}{l}\text { Coopera- } \\
\text { tivas }\end{array}$ & Mutuas & Asociaciones & $\begin{array}{c}\text { Empleo en la } \\
\text { ES }\end{array}$ & $\begin{array}{c}\text { Total } \\
\text { empleo** }^{*}\end{array}$ & $\%$ \\
\hline 1 & Holanda & 110.710 & n.d & 661.400 & 772.110 & 8.089 .071 & 9,5 \\
\hline 2 & Irlanda & 35.992 & 650 & 118.664 & 155.306 & 1.730 .381 & 9,0 \\
\hline 3 & Francia & 439.720 & 110.100 & 1.435 .330 & 1.985 .150 & 23.859 .402 & 8,3 \\
\hline 4 & Finlandia & 95.000 & 5.405 & 74.992 & 175.397 & 2.354 .265 & 7,5 \\
\hline 5 & Bélgica & 17.047 & 12.864 & 249.700 & 279.611 & 4.048 .499 & 6,9 \\
\hline 6 & Austria & 62.145 & 8.000 & 190.000 & 260.145 & 3.786 .969 & 6,9 \\
\hline 7 & Italia & 837.024 & n.d.* & 499.389 & 1.336 .413 & 21.477 .906 & 6,2 \\
\hline 8 & $\begin{array}{l}\text { Reino } \\
\text { Unido }\end{array}$ & 190.458 & 47.818 & 1.473 .000 & 1.711 .276 & 27.960 .649 & 6,1 \\
\hline 9 & Dinamarca & 39.107 & 1.00 & 120.657 & 160.764 & 2.684 .311 & 6,0 \\
\hline 10 & Alemania & 466.900 & 150.000 & 1.414 .937 & 2.031 .837 & 35.850 .878 & 5,7 \\
\hline 11 & España & 488.606 & 3.548 & 380.060 & 872.214 & 16.155 .305 & 5,4 \\
\hline 12 & Suecia & 99.500 & 11.000 & 95.197 & 205.697 & 4.252 .211 & 4,8 \\
\hline 13 & Eslovaquia & 82.012 & n.d. & 16.200 & 98.212 & 2.118 .029 & 4,6 \\
\hline 14 & Portugal & 51.000 & n.d.* & 159.950 & 210.950 & 4.783 .988 & 4,4 \\
\hline 15 & Estonia & 15.250 & n.d & 8.000 & 23.250 & 565.567 & 4,1 \\
\hline 16 & $\begin{array}{l}\text { Luxem- } \\
\text { burgo }\end{array}$ & 748 & n.d. & 6.500 & 7.248 & 187.809 & 3,9 \\
\hline 17 & Polonia & 469.179 & n.d. & 60.000 & 529.179 & 13.470 .375 & 3,9 \\
\hline 18 & R. Checa & 90.874 & 147 & 74.200 & 165.221 & 4.707 .477 & 3,5 \\
\hline 19 & Hungría & 42.787 & n.d & 32.882 & 75.669 & 3.831 .391 & 2,0 \\
\hline 20 & Grecia & 12.345 & 489 & 57.000 & 69.834 & 3.832 .994 & 1,8 \\
\hline 21 & Chipre & 4.491 & n.d & n.d & 4.491 & 307.305 & 1,5 \\
\hline 22 & Lituania & 7.700 & 0 & n.d & 7.700 & 1.378 .900 & 0,6 \\
\hline 23 & Eslovenia & 4.401 & 270 & n.d. & 4.671 & 888.949 & 0,5 \\
\hline 24 & Malta & 238 & n.d & n.d & 238 & 146.500 & 0,2 \\
\hline 25 & Letonia & 300 & n.d & n.d & 300 & 960.304 & 0,0 \\
\hline \multicolumn{2}{|c|}{ Total } & 3.663 .534 & 351.291 & 7.128 .058 & 11.142 .883 & 189.429 .435 & 5,9 \\
\hline \multicolumn{2}{|c|}{ Europa -15 } & & & & 10.233 .952 & 161.054 .638 & 6,4 \\
\hline \multicolumn{2}{|c|}{ Nuevos miembros -10} & & & & 908.931 & 28.374 .797 & 3,2 \\
\hline
\end{tabular}

n.d.: no disponible

n.d. : para Italia los datos de mutuas aparecen agregados con los de las cooperativas, y para Portugal con los de asociaciones.

**: Población ocupada entre 16 y 65 años, Eurostat, 2002.

Fuente: Chaves y Monzón (2007, pp. 48-49) 
identificar en la economía social un tipo de organizaciones que responden a criterios de doble circularidad, es decir, es a través de la participación en la toma de decisiones y en la implicación a la vida asociativa o cooperativa que los sujetos consiguen modificar las estructuras y por ende las instituciones formales.

Al mismo tiempo, las instituciones informales se ven modificadas para adaptar a los sujetos a un nuevo entorno, caracterizado por la experiencia económica del contrato dentro y fuera de la organización. De esta forma, aumentado los resultados económicos de los individuos, las organizaciones de economía social consiguen mejorar la posición de la personas como participantes, teniendo una posición con condiciones de ventaja frente a instituciones clásicas de los sistemas de la economía pública y la economía privada capitalista.

Es un hecho que en las últimas décadas - en contextos de crisis recurrentes-, el paradigma dominante se está transformando a "golpe de timón" gracias sobre todo a las propuestas de cambio estructural que ven como alternativa las organizaciones de economía social, al ser estas elementos del sistema económico con capacidad para valorizar las actividades económicas marginales que no fueron consideradas con interés por el sector privado y el público.

En estos últimos años, las instituciones europeas han publicado diversos documentos y han comunicado posiciones políticas sobre asuntos que ven implicada la economía social directa o indirectamente. Los documentos establecen una corriente que entiende que los objetivos de los tratados europeos pueden lograrse solamente a través de una mayor presencia de entidades de la economía social. ${ }^{7}$

Un segundo documento relevante en el proceso de legitimación de la economía social europea es la "Resolución sobre Economía Social” (Ciriec 20/2009, pp. 436-439), la cual fue presentada como Informe el 19 de diciembre del 2009 y fue aprobada por el Parlamento Europeo, por lo que establece un reconocimiento general del concepto de economía social, a nivel jurídico y a nivel estadístico, además de reconocer la importancia en la Estrategia de Lisboa.

Las premisas de la Resolución están basadas en el modelo social europeo y el paradigma social de la economía social acorde con los principios fundamentales del modelo. Hace hincapié en las consecuencias que se quieren alcanzar en cuanto a la política europea y valoriza la economía social al respecto. Es decir, la presencia de organizaciones de la economía social refuerza el modelo que los países miembros han decidido para la Unión Europea, tanto como identidad, como modelo de políticas europeas.

\footnotetext{
Resolución del Parlamento Europeo del 19 febrero del 2009 sobre Economía Social (conocido como Informe Toia). Dictamen del CESE 1454/2009 sobre las diversas formas de empresas. Comunicación de la Comisión sobre Fomento de las Cooperativas en Europa (COM, 2004). Dictamen Comité de las Regiones: "Asociaciones entre las autoridades locales y regionales y las organizaciones socioeconómicas: contribución al empleo, al desarrollo local y a la cohesión social"1 (2002/C192/13). DouE
} 12.08.2002 
Además, la Resolución expone la dimensión de la economía social que representa el $10 \%$ del total de las empresas y el $6 \%$ del empleo total. Las empresas son pequeñas y medianas empresas sostenibles, en las que las personas son más importantes que el capital. Aunque dentro del concepto se considera que aparte de "las cooperativas, las mutualidades, las asociaciones, las empresas y las organizaciones sociales y las fundaciones" existen otras formas existentes en cada Estado miembro, son comparables porque comparten las mismas características, por lo que la realidad social resulta ser más grande que los datos estadísticos oficiales. La Resolución considera que el modelo de empresa no se caracteriza por la dimensión sino más bien por los valores que comparten;

[...] la Es pone en evidencia un modelo de empresa que no se caracteriza por el tamaño o por sector de actividad sino por el respeto de valores comunes como la primacía de la democracia y de la participación de los actores sociales, de la persona y del objeto social sobre el beneficio individual; la defensa y la aplicación de los principios de solidaridad y de responsabilidad [...] (Parlamento Europeo, 2009, p. 5).

Por tanto, y aunque sea un modelo de empresa desconocido y criticado por enfoques teóricos parciales, la realidad es que la economía social necesita un mayor reconocimiento por parte de gobiernos nacionales y en el seno de la Unión Europea, sea tanto en su aspecto jurídico como estadístico, para dar visibilidad institucional y conocer la dimensión exacta del fenómeno social y económico.

Al tener en cuenta las premisas, la Resolución plantea diversas consideraciones generales sobre las funciones y necesidades de la economía social, tales como:

1. Papel esencial en la economía europea.

2. Objetivo de fortalecer la democracia industrial y económica.

3. Necesidad de reunir las condiciones previas de carácter político, legislativo y operativo adecuado, para prosperar como realidad social y económica.

4. Mismas normas sobre competencia que otras empresas, basado en un reconocimiento y un marco jurídico seguro para operar en igualdad a las otras empresas.

5. Un sistema económico que con un papel más importante reduciría el riesgo de especulación en los mercados financieros.

Un último documento que ha resultado relevante en el proceso de reconocimiento y legitimación a nivel europeo fue la Carta Abierta a la Comisión Europea del 13 noviembre del 2010, promovida por los profesores Carlo Borzaga (Borzaga et al., 2010), Jacques Defourny, Stefano Zamagni, Lars Hulgard, Rodger Spear, Adalbert Evers, Jerzy Hausner, Alberto Zevi y José Luis Monzón, además de estar subscrita por más de 400 investigadores y estudiosos. La carta es el resultado del discurso del comisario europeo del Mercado Interno, Michel Barnier. 
La carta, con el título "De las palabras a la acción: Apoyo a las empresas sociales y cooperativas para alcanzar una Europa más inclusiva, sostenible y próspera”, hace referencia al concepto utilizado por Michel Barnier en su discurso de posesión ante el Parlamento Europeo sobre el "modelo de economía social de mercado", el 13 de enero del 2010. El discurso del comisario Barnier consideró la prioridad de poner los ciudadanos al corazón del mercado europeo dando significado al concepto de mercados eficientes necesarios para el progreso humano; para ello se tendría en consideración el modelo de las sociedades cooperativas y las mutuales, además de las fundaciones y las actividades caritativas, dirigido a la concepción del acto de negocio social (social business act) que podría ser orientado al negocio que obtiene beneficios y lucha contra la exclusión social (Barnier, 2010).

Los académicos signatarios de la carta consideran el discurso del comisario Barnier como un acto para promover la identidad de la economía social en el desarrollo socioeconómico de la Unión Europea entre figuras relevantes a nivel político, académico y profesional. La razón fundamental que sostiene la carta es la importancia que ha tenido la presencia y el desarrollo de entidades de economía social en las últimas décadas. Estas entidades han participado en la producción de bienes y servicios de gran interés general y han favorecido el desarrollo económico y la cohesión social. La carta se ha convertido en una acción que refuerza el interés de las instituciones europeas.
El objetivo de la carta está relacionado con la necesidad existente para fomentar una línea de trabajo que haga emerger la economía social como agente económico y social a nivel institucional junto con la economía pública y la privada capitalista. Los objetivos específicos son claros y apuestan por una acción plural de lobbying europeo dirigida a matizar y evidenciar que el concepto de economía social tiene el consenso de la comunidad académica y profesional.

La carta, en específico, resalta diversos puntos que sirven para establecer una ruta de trabajo de las instituciones públicas europeas. Estos son:

1. Existe una necesidad de refundar el papel tradicionalmente asignado a los diferentes tipos de empresas.

2. Reconocimiento de las mutualidades y las cooperativas como ejemplos de organizaciones de economía social.

3. Valorización de empresas de economía social para contribuir a la economía europea y su crecimiento, haciendo compatibles los objetivos económicos y sociales.

4. Legitimación a nivel internacional de los negocios sociales (social business) como un universo de organizaciones que representan la innovación social europea y que se reconoce e imita en todo el mundo.

5. Función relevante y fundamental en la actual crisis económica de las entidades de economía social.

6. Escasa atención de los políticos europeos en las últimas décadas por las entidades de economía social. 
Los puntos representan los trabajos de estudio y análisis del fenómeno en estas últimas décadas, y son la base de una serie de recomendaciones para establecer una acción de políticas públicas a favor de la economía social. El mismo título de la carta tiene una intención constructiva entre el mundo académico-profesional y la función pública de los hacedores de políticas. Por tanto, la función de la carta se ubica en un momento de crisis actual que cuestiona el modelo económico tradicional y se muestra necesaria como acción, a fin de incentivar un proceso político que fomente y legitime la presencia de la economía social en las políticas económicas a través de una estructura de políticas públicas favorable.

Las recomendaciones que se definen en la Carta se pueden sintetizar en una serie de políticas concretas para toda la Unión Europea. Son las siguientes:

1. Adopción de políticas fiscales que reconozcan la especificidad de las entidades de economía social.

2. Definición de normas de contratación pública que consideran el valor añadido para los objetivos de la Unión Europea (cohesión e inclusión social).

3. Definición de políticas industriales y económicas que apoyen la creación y el desarrollo de organizaciones de economía social.

4. Uso de los fondos estructurales para fomentar la formación y el desarrollo de organizaciones de economía social.

5. Apoyo a la investigación y la creación de conocimiento en economía social.
El proceso de acuerdo sobre la legitimación entre el mundo académico y político demuestra el interés mutuo por la transformación social a través de la promoción y el fomento de un tipo de empresa y un estilo de emprendimientos basados en principios de solidaridad y democracia económica. El proceso que se ha visto en el contexto europeo por parte del mundo académico y el sector desde el 2009 ha sido incremental respecto a la importancia y el reconocimiento de la economía social, como ejemplo la Primera Semana Europea de las Cooperativas, organizada por diferentes organizaciones cooperativas europeas entre el 23 y el 27 de abril del 2012 (Aciamericas, 2012), acto que rebatió la importancia del modelo cooperativo para alcanzar los objetivos de la Unión Europea, además de conseguir un crecimiento económico sostenible e incluyente socialmente.

\section{Democratizar la economía, una apuesta de la economía solidaria colombiana desde la innovación social}

La economía solidaria en Colombia está caracterizada por la presencia de cooperativas, mutuales y fondos de empleados, pero no sólo estos. Si consideramos las dos normas fundamentales del ordenamiento jurídico de Colombia, Ley 79 del 1988 y Ley 454 del 1998, el cuadro de tipologías de organizaciones con valores basados en la economía solidaria sería el que se observa en la tabla 4. 
Tabla 4. Estructura organizacional del sector solidario en Colombia

\begin{tabular}{|c|c|c|c|c|}
\hline & $\begin{array}{l}\text { Movimiento } \\
\text { cooperativo }\end{array}$ & Sector cooperativo & $\begin{array}{c}\text { Sistema de economía } \\
\text { solidaria }\end{array}$ & $\begin{array}{l}\text { Sector social y } \\
\text { solidario }\end{array}$ \\
\hline 氙芯 & Ley 79 de 1988 & Ley 79 de 1988 & Ley 454 de 1998 & $\begin{array}{l}\text { Propuestas de un } \\
\text { derecho solidario. }\end{array}$ \\
\hline 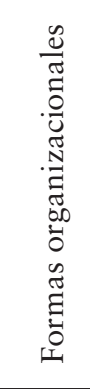 & $\begin{array}{l}\text { Cooperativas } \\
\text { de producción, } \\
\text { consumo, mixtas } \\
\text { y de integración. }\end{array}$ & $\begin{array}{l}\text { Movimiento coope- } \\
\text { rativo + instituciones } \\
\text { auxiliares del } \\
\text { cooperativismo y } \\
\text { precooperativas. }\end{array}$ & $\begin{array}{l}\text { Sector cooperativo + } \\
\text { sociedades mutuarias } \\
\text { (fondos de empleados y } \\
\text { asociaciones mutualistas) } \\
\text { + empresas comunitarias } \\
\text { + empresas asociativas } \\
\text { de trabajo + empresas } \\
\text { solidarias de salud + } \\
\text { administraciones públicas } \\
\text { cooperativas. }\end{array}$ & $\begin{array}{l}\text { Sistema de eco- } \\
\text { nomía solidaria }+ \\
\text { organizaciones de } \\
\text { interés general. }\end{array}$ \\
\hline 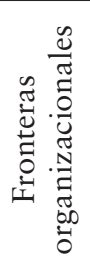 & $\begin{array}{l}\text { Mutuales, fondos } \\
\text { de empleados, } \\
\text { instituciones } \\
\text { auxiliares del } \\
\text { cooperativismo, } \\
\text { precooperativas. }\end{array}$ & $\begin{array}{l}\text { Sociedades mutuarias } \\
\text { (fondos de empleados } \\
\text { y asociaciones mutua- } \\
\text { listas) y empresas de } \\
\text { servicios en las formas } \\
\text { de administraciones } \\
\text { públicas cooperativas. }\end{array}$ & $\begin{array}{l}\text { Organizaciones de interés } \\
\text { general: juntas de acción } \\
\text { comunal, fundaciones, } \\
\text { corporaciones, redes de } \\
\text { voluntariado, cajas de } \\
\text { compensación familiar. }\end{array}$ & $\begin{array}{l}\text { Organizaciones } \\
\text { sindicales, } \\
\text { sacramentales y } \\
\text { políticas de carác- } \\
\text { ter partidista. }\end{array}$ \\
\hline
\end{tabular}

Fuente: Álvarez y Serrano, 2006, p. 188

Las diversas entidades de la economía solidaria colombiana ejercen su función económica y social en un escenario cambiante y muy dinámico, con altos índices de competitividad gracias a la gobernabilidad de los factores. El aporte de Serrano (2007a) nos indica cómo la economía solidaria colombiana, en el caso de las cooperativas, para ser competitivas deben ofrecer servicios al menor costo posible y en condiciones de calidad, oportunidad y flexibilidad, lo que está relacionado directamente con el cumplimiento con eficacia y eficiencia de sus objetivos.

La evolución de las entidades de economía solidaria colombiana —como hemos visto en los apartados anterio- res- está basada en una alta presencia en el contexto socioeconómico, no sólo por el número de entidades reconocidas, sino también por el incremento gradual tanto de asociados como de empleados. Esto nos lleva a considerar que han sabido mantener la unión fundamental de los valores democráticos con los principios cooperativos regulando su actividad productiva y de gobernabilidad. De hecho, han conseguido definir los mecanismos de distribución de la riqueza, corregir los desequilibrios del mercado del trabajo, reforzar las instituciones democráticas presentes en el territorio y, por tanto, promover la democracia económica, con principios de justicia y equidad, fundamentales para promover la cohesión 
social, gracias a "la capacidad de generar una dinámica relacional que les permite la utilización plena del factor humano y de los recursos físicos y financieros" (Serrano, 2007a, p. 4).

La necesidad actual de valorar las organizaciones de economía solidaria colombiana implica reconocer la identidad de conjunto de la economía social y solidaria desde la articulación y ordenación de sus prioridades, las cuales no están relacionadas con la racionalidad económica sino más bien con la relación simpatética entre individuos. La identidad de la dinámica relacional especial de las mismas sociedades industriales ha ido incrementando su sensibilidad hacia la cohesión social. Los valores y principios cooperativos han comenzado a adentrarse paulatinamente en el mismo discurso, alejándose de la visión de la económica neoclásica ortodoxa que consideraba los valores fuera de la esfera de la ciencia económica (Ben-Ner y Putterman, 1999).

Las entidades de la economía social y solidaria tienen relevancia no sólo por la cantidad sino también por la calidad de los bienes y servicios que producen y que se corresponden con las necesidades cambiantes de las instituciones económicas y sociales, y también por la necesidad de nuevas formas y modelos de gobernabilidad de los factores productivos en mercados que mantienen y promueven la competitividad global de economías territoriales.

Si se considera la definición de gobernabilidad de la cooperativa basada en el desarrollo de la capacidades para dirigir y conducir el rumbo de la empresa hacia el logro de sus propósitos y objetivos (Serrano, 2007a), nos resulta altamente interesante observar cómo la ventaja de la economía social y solidaria está basada en la ventaja cooperativa (Silva y Dávila, 2002). La capacidad de generar un paradigma propio y exclusivo basado en la misma filosofía de la gestión estratégica de la función cooperativa hace que la cooperativa — como empresa ideal tipo de la Ess- obtenga una ventaja comparativa frente a las empresas que basan su función en la maximización del capital a través de la instrumentalización del factor trabajo, tecnológico y ambiental.

La ventaja cooperativa está, por tanto, basada en la maximización del bienestar de los trabajadores a través de condicionar el capital y el factor tecnológico, salvaguardando y respetando el factor ambiental como elemento de territorialidad y el arraigo de la Ess a esta.

La existencia de propiedades y atributos que miran a democratizar la economía, no salva de los riesgos y peligros que la Ess pueda sufrir lo que se denomina deriva isomórfica, así como se preguntó el profesor Rymel Serrano durante el discurso inaugural de la I Conferencia del Ciriec-Colombia (anteriormente citado). Así pues, se hace necesario reflexionar cómo las cooperativas pueden ser actores con capacidad de crear un orden social nuevo, orientado en la persona.

La alta competitividad del mercado hace que las entidades de Ess puedan incurrir en riesgo moral frente a los aso- 
ciados, empleados y el territorio donde realizan su actividad económica. Rymel Serrano ofrece una importante contribución al respecto. El profesor Serrano (2007b) identifica tres tendencias que son clasificadas como peligrosas en América Latina, debido al notable crecimiento y la expansión acelerada de estas. Esta situación podría afectar a su gobernabilidad genuina poniendo en peligro la participación de sus asociados (Serrano, 2007a). Los tres peligros que identifica el autor están referidos a:

1. La función comercial orientada a la conquista de nuevos mercados.

2. La concentración de poder en grupos de expertos y técnicos que conforman el aparato técnico-burocrático.

3. La dependencia excesiva de los centros oficiales de poder (paternalismo).

Por tanto, es fundamental identificar los escenarios que fortalezcan la ventaja comparativa de la Ess en su conjunto, con el fin de formular respuestas sistémicas para alcanzar el éxito del ess en un espacio eurolatinoamericano, desde una perspectiva de innovación social. En el caso de las cooperativas, la relación entre la innovación y el éxito cooperativo es relativa, ya que se innova y se tiene éxito en un contexto dado y con unas condiciones particulares (Internan, citado en Dávila, 2004). Ramírez (2002) identifica el liderazgo y la participación como elementos que alimentan el modo de gestión, la cual es una característica que hace distinguir la estrategia cooperativa (Ramírez, 2002).
Es en este sentido, los elementos que inciden en el éxito son la toma de decisiones y el impacto en "la generación de un estímulo a la creatividad individual en un marco colectivo orientado por el afán de satisfacer al asociado [...] la toma de decisiones consensuada en el marco participativo impulsa la generación de nuevas ideas" (Dávila, 2004 p. 48).

La innovación desde el enfoque de la Ess se puede definir como la formulación de un paradigma de participación y liderazgo extendido a todos los stakeholder que cooperan en pro del beneficio mutuo, convirtiéndose en una organización multistakeholder.

La gestión de la estrategia cooperativa tiene como objetivo encontrar las soluciones más satisfactorias para el mayor número de individuos. En concreto, se contrapone con el concepto de eficiencia social del libertarismo utilitarista, en el que la función sumatoria de las utilidades de los individuos es considerada como elemento principal para determinar el cálculo racional. En cambio, la organización "cooperante" propone formas para el cálculo de la eficiencia social, en la cual el elemento determinante de la combinación de las utilidades de los individuos está basado en la función multiplicadora de las utilidades. Por tanto, en el caso de que un individuo obtenga una utilidad cero, la utilidad final (agregada) será igual a cero, obligando al grupo como organización a elaborar estrategias desde la participación paritaria de los miembros de la organización. 
Así pues, el éxito de la organización cooperativa reside en la capacidad de establecer un "posicionamiento cooperativo" por parte de todos aquellos stakeholder que participan en la multidimensionalidad de la organización, tanto interna como externamente. Para ello es necesario un marco conceptual (Ramírez, 2002) y cognitivo favorecedor, que tenga como objetivo central la participación y empoderamiento de los stakeholder en la formulación de la estrategia cooperativa en un marco colectivo orientado por la toma de decisiones consensuadas a través de las generación de nuevas ideas.

Una conclusión que emerge desde la teoría cooperativa es la capacidad que las organizaciones deben tener para establecer congruencia y coherencia entre los principios y valores cooperativos y los resultados obtenidos a través de la formulación de una estrategia sistémica. El riesgo de los costes de incoherencia puede incidir en la identidad cooperativa original; los cuales se concentrarán en la percepción y las expectativas futuras condicionando la utilización de la forma organizativa por parte de los asociados, lo que llevaría a: (I) desvirtuar el origen de la cooperación; (II) instrumentalizar la organización en busca de una mayor optimización de rentas individuales a menoscabo de la identidad del colectivo.

Así pues, el acto de innovar en la cooperativa se basa en generar dinámicas fuertes y coherentes comprometidas con los objetivos participativos de la organización, sin caer en la demagogia utópica de un modelo que puede resolver a priori los problemas y necesidades de un grupo de personas, sino más bien posicionar sólidamente la ventaja cooperativa como elemento competitivo de maximización de utilidades colectivas frente a organizaciones gestionadas sólo para maximizar las utilidades individuales.

\section{Nuevos escenarios de innovación social a través de alianzas publico-privadas para el fomento de la Ess en un espacio eurolatinoamericano}

La innovación social es una nueva combinación o configuración de prácticas sociales en ciertas áreas de acción o en contextos sociales definidos por los actores o por una constelación de actores con el objetivo de satisfacer o responder a necesidades y problemas (Howaldt y Schwarz, 2010, p. 21).

La Ess considera la empresa como "un sistema social abierto en constante interacción con la sociedad" (Dávila, 2004, p. 30), en la cual se tejen relaciones sociales entre agentes internos y externos a la actividad de la organización. El elemento común de todas la organizaciones cooperativas es la "conjunción de la asociación de personas con la empresas económica, que tiene un doble objetivo económico y social, donde el excedente económico es el medio y no el fin y se distribuye al asociado de acuerdo con el uso de los servicios" (Dávila, 2004, p. 30). Las organizaciones de la Ess establecen mecanismos de innovación social, ya que, para responder a sus necesidades, los actores crean formas de 
gestión de acuerdo con la ordenación de factores productivos en modo genuino y auténtico.

Aunque existen motivaciones sociales, la razón por la cual las personas se unen en una asociación de carácter cooperativo es en última instancia económica (Arango et al., 2005), transfiriendo valores de solidaridad, asociación y cooperación entre las mismas personas que han aplicado la racionalidad económica para asociarse, dando como resultado la presencia en economías de mercado competitivas, abiertas y altamente dinámicas. El estudio de la Fundación para la Educación Superior y el Desarrollo (Fedesarrollo) sobre la economía solidaria, identifica el beneficio de la ayuda mutua para los asociados al momento de crear o asociarse a una empresa solidaria (Arango, et al., 2005). Si la creación es en última instancia económica, es por tanto necesario identificar las motivaciones que hacen permanecer a los asociados una vez obtenido el servicio o la satisfacción de la necesidad, y a su vez identificar cómo se fomentan nuevas fases de innovación desde adentro de las organizaciones, lo cual constituye un tema de estudio e investigación relevante para determinar el comportamiento socioeconómico de las organizaciones de la economía social y solidaria en un espacio eurolatinoamericano.

El 2012 es un punto de inflexión, sea por la celebración del Año Internacional de las Cooperativas (ONU), sea por el VII Encuentro de la Sociedad Civil Organizada Unión Europea-América Latina. La ocasión es propicia para entablar un diá- logo sobre el concepto dual de economía social y solidaria, sobre todo si el diálogo se construye en torno a la constitución y formación de redes que den origen a proyectos eficientes, orientados a la intercooperación de las organizaciones de la ess junto con la cooperación internacional europea. Como se plantea en el dictamen del Comité Económico y Social Europeo sobre el tema de la "Economía social en América Latina” (Chaves Ávila y Monzón Campos, 2007), la cooperación a través de planes empresariales de la economía social y solidaria para el desarrollo sostenible puede ser fundamental para caracterizar los intercambios comerciales en un espacio internacional por parte de actores que pueden consolidar un interés comercial basado en el conjunto de identidades comunes a las dos orillas del Atlántico.

\section{Conclusiones}

Debido a la escasa regulación del sector en sus aspectos más específicos, y la baja participación en el modelo de colaboración pública-privada dirigido a la planificación e implementación de estrategias de competitividad, no encontramos gran reconocimiento de la importancia de la ESS en el espacio eurolatinoamericano. Del mismo modo, el modelo de colaboración pública-privada ha sido influenciado por los ciclos políticos (Devlin y Moguillansky, 2010), produciendo una incapacidad de gobernanza del sector solidario en las políticas públicas. El sector debe entender y construir su capacidad de gobernanza en la esfera 
pública, es decir, establecer los procesos mediante los cuales "los actores decidan sus objetivos de convivencia y las formas de coordinarse para realizaros; su sentido de dirección y su capacidad de dar dirección” (Aguilar, 2008, p. 90), en las dos orillas del Atlántico.

La necesidad del sector en la participación de alianzas publico-privadas debe estar orientada a construir un escenario positivo, que valorice y legitime la Ess. De igual modo, es necesaria una mayor atención como disciplina de estudio, como actor estratégico en la formulación de políticas públicas sectoriales y transversales y no como mero observador de los cambios sociales.

Asimismo, es necesario formular una agenda de políticas públicas que entienda la identidad de la economía social y solidaria, además de considerar las sinergias $\mathrm{y}$ fortalezas que ponen en valor la gobernanza a través de alianzas con organismos públicos, orientadas a "la coordinación, elaboración, supervisión, regulación, elaboración y diseño de mecanismos y estrategias que permitan un desarrollo eficaz de la política postulada" (DNP, 2010, p. 33) y al "establecimiento de programas de acompañamiento y asistencia técnica para los emprendedores en el marco de políticas activas de empleo" (Nilsson, 2012, p. 10).

La oportunidad del Año Internacional de las Cooperativas es una ventana importante para tratar el tema desde diferentes miradas. La economía social y solidaria tiene el reto de demostrar su válidez frente los cambios sociales, a través de la práctica de la solidaridad, cooperación, autoayuda y democracia económica como elementos claves para el éxito del modelo.

\section{Referencias}

Aciamericas. (2012, 6 de mayo). Bruselas fue sede de la Semana Europea de las Cooperativas que conmemoró AIC. Recuperado de http://www.aciamericas.coop/ Bruselas-fue-sede-de-la-Semana

Aguilar Villanueva, L. (2008). Gobernanza y gestión publica. México: Fondo de Cultura Económica.

Álvarez Rodríguez, J. y Serrano Uribe, R., (2006). Estructuras de integración del cooperativismo y la economía solidaria en Colombia. Revista Venezolana de Economía Social, Año 6, (11), 184-201.

Arango, M., et al. (2005). Reflexiones sobre el aporte social y económico del sector cooperativo colombiano (Vol. 15). Bogotá: Fedesarrollo.

Barnier, M. (2010). Speech at the European Parlament, 13th January. Recuperado de http:// ec.europa.eu/commission_2010-2014/ barnier/docs/speeches/20100113/ imco_en.pdf

Bruni, L. y Zamagni, S. (2004). Economia Civile. Efficienza, equitá, felicitá pubblica. Bologna, Italia: Ed. Il Mulino

Ben-Ner, A. y Putterman, L. (1999). Valores e instituciones en el análisis económico. Revista de Economía Pública, Social y Cooperativa, (33), 43-77.

Borzaga, C. et al. (2010). Carta Abierta a la Comisión Europea: "De las palabras a la acción: apoyo a las empresas sociales y cooperativas para alcanzar una europa más inclusiva, sostenible y próspera". [Version en inglés]. Recuperado de http://www. euricse.eu/sites/default/files/db_uploads/ documents/1279034974_n558.pdf. 
Castillo Sandoval, D. et al. (2011). Políticas Públicas y cooperativismo en Colombia. 30 años de encuentros y desencuentros. Bogotá: IEMP Ediciones.

Chaves Ávila, R. y Monzón Campos, J. (Coord.) (2007). Informe sobre la situación de la Economía Social en Europa. Presentado el 6 de diciembre del 2007 en el Consejo Económico y Social dell'UE. Recuperado de http://www. eesc.europa.eu/?i=portal.en.publications. 83

Ciriec. (2009). Resolución sobre economía social.

Confecoop. (2012). Desempeño Sector Cooperativo Colombiano 2011. (Confecoop, Ed.) Recuperado de http://www.confecoop.coop/ index.php?option $=$ com_content\&view=arti cle\&id=247\&Itemid $=529$

Congreso de la República. Ley 79 de 1988. Por la cual se actualiza la Legislación Cooperativa. Diario oficial, 38648, 10 de enero de 1989. Bogotá.

Congreso de la República. Ley 454 de 1998. Por la cual se determina el marco conceptual que regula la economía solidaria, se transforma el Departamento Administrativo Nacional de Cooperativas en el Departamento Administrativo Nacional de la Economía Solidaria, se crea la Superintendencia de la Economía Solidaria, se crea el Fondo de Garantías para las Cooperativas Financieras y de Ahorro y Crédito, se dictan normas sobre la actividad financiera de las entidades de naturaleza cooperativa y se expiden otras disposiciones. Diario oficial, 43357.4 de agosto de 1998. Bogotá.

Dávila Ladrón de Guevara, R. (2004). La cooperativa, una forma organizacional y administrativa particular. Bogotá: Pontificia Universidad Javeriana.

Departamento Nacional de Planeación (DNP). (Febrero, 2010). Conpes 3639-Política de desarrollo empresarial para el sector de la economía solidaria. Recuperado de http:// www.dnp.gov.co

Devlin, R. y Moguillansky, G. (2010). Alianzas público-privadas para una nueva visión estratégica del desarrollo. Santiago de Chile: Naciones Unidas-Cepal-Secretaría General Iberoamericana.

Gaiger, L. I. (2009). Emprendimientos económicos solidarios. En Cattani, A, Coraggio, J. y Laville, J. Diccionario de la otra economía (p. 381). Buenos Aires: Altamira.

Howaldt, J. y Schwarz, M. (2010, mayo). Social Innovation: Concepts, research fields and international trends. Recuperado el 24 de abril del 2012 de Imo-International Monitoring: http://www.internationalmonitoring.com

Monzón, J. L. y Defourny, J. (1992). Economía Social. Entre economía capitalista y economía pública. Valencia: Ciriec-España.

Mutuberría, V. y Serrano, A. (septiembre, 2010). Hacia otra economía en América Latina: El papel de la Economía Social. Congreso Internacional: Actas del XIV Encuentro de Latinoamericanistas Españoles. Santiago de Chile. pp. 2953-2989.

Nilsson, S. (2012). Dictamen del Comité Económico y Social Europeo sobre el tema "La Economía social en América Latina". Recuperado de www.eesc.europea.eu

Parlamento Europeo (2009). Informe sobre economía social. Recuperado de http:// www.europarl.europa.eu/sides/getDoc. do? pubRef=-//EP//NONSGML+REPORT+A62009-OOI 5+O+DOC+PDF+VO//ES

Ramírez, L. (2002). Fundamentos de gestión cooperativa en procesos de formulación estratégica: La ventaja cooperativa. Sherbrooke, Canada: Irecus-Universidad de Sherbrooke.

Salamon, L. y Anheier, H. (1997). Defining the Nonprofit Sector: A Cross-national Analysis. Manchester and Nueva York: Manchester University Press.

Sánchez Álvarez, C. (2011). La legitimación internacional de la Economía Social. 2012-Hacia el Año Internacional de las Cooperativas. En C. Spain. Third International Research Conference. Valencia: Ciriec-España. 
Serrano Uribe, R. (2007a). La gobernabilidad en las cooperativas. Notas de investigación. Recuperado de www.indesco.coop

Serrano Uribe, R. (2007b). La democracia en las cooperativas. Revista Cooperativismo y Desarrollo (91). Recuperado de www. dansocial.gov.co
Silva J. y Dávila Ladrón de Guevara, R. (Comp.) (2002). Gestión y desarrollo: El caso de la cooperativa en Colombia. Bogotá: Pontificia Universidad Javeriana.

Tratado de la Unión Europea (TUE) (febrero, 1992). s. d. 